\title{
Spermidine Prevents Heart Injury in Neonatal Rats Exposed to Intrauterine Hypoxia by Inhibiting Oxidative Stress and Mitochondrial Fragmentation
}

\author{
Nannan Chai $\mathbb{D}^{1,2}$ Hao Zhang, ${ }^{1,3}$ Lingxu Li, ${ }^{1}$ Xue Yu, ${ }^{1}$ Yan Liu, ${ }^{4}$ Yan Lin, ${ }^{5}$ Lina Wang, \\ Jiamin Yan, ${ }^{6}$ Sazonova Elena Nikolaevna $\mathbb{D}^{7},{ }^{7}$ and Yajun Zhao ${ }^{1}{ }^{1}$ \\ ${ }^{1}$ Department of Pathophysiology, Harbin Medical University, Harbin 150086, China \\ ${ }^{2}$ Department of Nursing, Medical School of Chifeng University, Chifeng 024000, China \\ ${ }^{3}$ Pathology Department, First Affiliated Hospital of Soochow University, Suzhou 215006, China \\ ${ }^{4}$ Department of Biochemistry, Harbin Medical University, Harbin 150086, China \\ ${ }^{5}$ Department of Pathophysiology, Qiqihar Medical University, Qiqihar, Heilongjiang 161006, China \\ ${ }^{6}$ Laboratory Center of Molecular Biology, Harbin Medical University, Harbin 150086, China \\ ${ }^{7}$ Department of Physiology, Far Eastern State Medical University, 680000, Russia
}

Correspondence should be addressed to Sazonova Elena Nikolaevna; sazen@mail.ru and Yajun Zhao; zhaoyajun1964@163.com

Received 22 November 2018; Revised 14 March 2019; Accepted 23 April 2019; Published 14 May 2019

Academic Editor: Gerardo García-Rivas

Copyright (C) 2019 Nannan Chai et al. This is an open access article distributed under the Creative Commons Attribution License, which permits unrestricted use, distribution, and reproduction in any medium, provided the original work is properly cited.

\begin{abstract}
Intrauterine hypoxia (IUH) is a common intrauterine dysplasia that can cause programming of the offspring cardiovascular system. In this study, we hypothesized that placental treatment with spermidine (SPD) can prevent heart injury in neonatal offspring exposed to IUH. Pregnant rats were exposed to $21 \% \mathrm{O}_{2}$ or $10 \% \mathrm{O}_{2}$ (hypoxia) for 7 days prior to term or were exposed to hypoxia and intraperitoneally administered SPD or SPD+difluromethylornithine (DFMO) on gestational days 15-21. Seven-day-old offspring were then sacrificed to assess several parameters. Our results demonstrated that IUH led to decreased myocardial ornithine decarboxylase (ODC) and increased spermidine/spermine $\mathrm{N}^{1}$-acetyltransferase (SSAT) expression in the offspring. IUH also resulted in decreased offspring body weight, heart weight, cardiomyocyte proliferation, and antioxidant capacity and increased cardiomyocyte apoptosis and fibrosis. Furthermore, IUH caused mitochondrial structure abnormality, dysfunction, and decreased biogenesis and led to a fission/fusion imbalance in offspring hearts. In vitro, hypoxia induced mitochondrial ROS accumulation, decreased membrane potential, and increased fragmentation. Notably, all hypoxia-induced changes analyzed in this study were prevented by SPD. Thus, in utero SPD treatment is a potential strategy for preventing IUH-induced neonatal cardiac injury.
\end{abstract}

\section{Introduction}

Newborns with intrauterine growth restriction (IUGR) often experience adverse perinatal outcomes that present with an increased mortality risk. The clinical evidence of cardiovascular dysfunction in fetal and/or early neonatal life supports the notion of perinatal programming before the onset of significant cardiovascular disease (CVD) in adulthood [1,2]. Intrauterine hypoxia (IUH) is the most common adverse intrauterine condition and occurs under various circumstances such as high-altitude pregnancy [3], preeclampsia, placental insufficiency, and any inflammatory condition during pregnancy caused by gestational diabetes or even maternal obesity [4]. A number of studies have reported that oxidative stress is the basis of fetal complications associated with low birth weight and developmental plasticity, excessive generation of reactive oxygen species (ROS), and/or a decrease in antioxidant defense, leading to indiscriminate damage to the developing fetus-all molecular mechanisms implicated in fetal programming of CVD [5]. Fetal hearts of pregnant rats suffering from prenatal hypoxia developed oxidative stress at the end of pregnancy, 
after which the offspring developed impaired peripheral artery relaxation and altered heart contractility in adulthood [6]. Mitochondria are the main organelles involved in the production and regulation of ROS. IUGR leads to increased oxidative stress in offspring rat hepatic mitochondria and impaired hepatic mitochondrial function [7]. A similar result was found in the pancreases of IUGR rat offspring [8]. More recently, a study showed that cardiac mitochondrial respiratory function was impaired in guinea pig offspring exposed to IUH [9]. A balance between mitochondrial fusion and fission is necessary to maintain normal mitochondrial morphology, number, and function in the heart [10]. Song et al. [11] revealed that cardiac-specific abrogation of either mitochondrial fusion (mitofusin 1 (Mfn1) and Mfn2) or fission (Drp1 ablation) in adult mouse hearts provoked lethal cardiac pathology. Papanicolaou et al. [12] further showed that transgenic mice deficient in cardiac-specific MFN1 and MFN2 starting from the late embryonic period displayed severe mitochondrial dysfunction on the 7th day after birth, developed cardiomyopathy, and all died within 14 days. However, much is yet to be discovered regarding the effects of prenatal hypoxia on neonatal cardiac mitochondrial dynamics and function.

Polyamines (PAs) include spermine (SP), spermidine $(\mathrm{SPD})$, and their precursor putrescine (PU) and are present in all types of mammalian cells. Intracellular levels of PAs are maintained and tightly controlled by enzymes that catalyze rate-limiting steps of their biosynthesis by ornithine decarboxylase (ODC) and catabolism by spermidine/ spermine-N1-acetyltransferase (SSAT) [13]. Polyamines are small polycations essential for all cellular life and are involved in gene expression [14], cell growth and differentiation [14], anti-inflammatory effects [15], antiapoptosis [16], protection against oxidative stress [17, 18], induction of autophagy [19], stabilization of cell and mitochondrial membranes [20], and embryonic development $[21,22]$. Both SP and SPD can neutralize a wide spectrum of ROS including $\mathrm{H}_{2} \mathrm{O}_{2}$ [23], $\mathrm{O}_{2}{ }^{--}$[24], and $\mathrm{HO}^{\circ}[25,26]$ as well as singlet oxygen [27]. We previously demonstrated that exogenous PAs reduce myocardial ischemia/reperfusion injury by inhibiting the production of ROS and opening of the mitochondrial permeability transition pores (mPTP) [28]. PAs can inhibit the $\mathrm{H}_{2} \mathrm{O}_{2}$-induced decrease in mitochondrial respiratory function in a concentration-dependent manner in cardiomyocytes [29]. It is noticeable that polyamine levels in the placental tissue of the sheep exposed to IUH were found decreased [21], while supplementation with exogenous PAs ameliorated embryonic dysplasia [22]. A recent study showed that L-arginine (Arg), a precursor of PA synthesis, is essential during pregnancy for growth and development of the conceptus and dietary supplementation of Arg during gestation was effective in improving embryonic survival and development of the conceptus in many species including humans, pigs, sheep, mice, and rats [30]. However, it is unclear whether administration of exogenous PAs to hypoxic mothers during pregnancy can reduce myocardial injury in newborn offspring.

Emerging evidence implicates that the important role of SPD in disease and health has received more attention recently [31, 32]. SPD, but not SP, is essential for early development and organogenesis [33], and SPD can significantly alleviate lipopolysaccharide-triggered inflammation in a zebrafish model [15]. Jamwal and Kumar revealed that rats administered with SPD at $5-10 \mathrm{mg} / \mathrm{kg} /$ day for 21 days intraperitoneally exerted neuroprotective effect in Huntingtonlike neuropathologies [34]. SPD (5 mg/kg ip) treatments in mice for $10 \mathrm{~d}$ led to a partial rescue of histological alterations in muscle defects [35]. Accordingly, $5 \mathrm{mg} / \mathrm{kg} /$ day SPD was selected to be given to the hypoxic mother during the late stage of pregnancy in order to study the protection of exogenous SPD on myocardial injury in newborn offspring suffered from maternal hypoxia.

\section{Materials and Methods}

2.1. Experimental Animals. All experiments were carried out following the National Institutes of Health guidelines, and all procedures were approved by the Local Ethics Review Committee of Harbin Medical University (China). Male and female Wistar rats (3 months old) were purchased from Harbin Medical University Experimental Center. Rats at a female: male ratio of 2:1 were randomly mated in one cage and tested for pregnancy via a vaginal smear obtained the following morning, which was examined for the presence of sperm, or through a copulatory plug found in the vagina, which signified day 0 of pregnancy. The pregnant rats were housed in rooms with controlled humidity (60\%), controlled temperature $\left(21^{\circ} \mathrm{C}\right)$, and a $12: 12 \mathrm{~h}$ light-dark cycle.

2.2. Intrauterine Hypoxia Model. From day 15 to day 21 of pregnancy, the hypoxic rats $(n=9)$ were placed inside a plexiglass chamber with a maternal oxygen supply of $10 \%$ for $4 \mathrm{~h}$ every day. The percentage of oxygen in the plexiglass chamber was monitored by continuous infusion of a nitrogen gas and air mixture with an oxygen analyzer (Pro OX120; BioSpherix, New York, NY). We placed calcium chloride into the chamber to absorb excess carbon dioxide and water vapor for humidity control to avoid carbon dioxide retention and acidosis. In the hypoxic group, femoral artery intubation was performed in pregnant rats before exposure to hypoxia. The catheter consists of an artery catheter, a connecting tube, and a three-way valve. After successful intubation, the incision was covered with gauze. Then, the pregnant rats were exposed to hypoxia. During hypoxia, we adjusted the three-way valve to communicate with the syringe, blood was collected once every hour, and the collected blood was immediately dripped onto the blood gas testing chip (EG7+; Abbott, Lake Bluff, IL). The chip was inserted into the portable blood gas analyzer (i-STAT 200; Abbott); after which, the partial pressure of oxygen, blood oxygen saturation, and $\mathrm{PH}$ were measured. Arterial oxygen partial pressure of pregnant rats was maintained at $50-55 \mathrm{mmHg}$, and blood oxygen saturation was maintained at $80-85 \%$ [36-38]. This experimental procedure was carried out as a preexperiment to successfully establish our rat model of IUH. Prior to term (22 days), SPD or SPD+difluoromethylornithine (DFMO; an inhibitor of the key PA synthesis enzyme ODC) were administered intraperitoneally (ip) to 
the hypoxic rats for 7 days (gestational days 15-21). The control rats were maintained at room air conditions $(21 \%$ $\mathrm{O}_{2}$ ) throughout pregnancy. The animals were randomly divided into four groups: (1) normoxic control group, control mother $+0.9 \%$ saline $(1 \mathrm{~mL} / \mathrm{kg} / \mathrm{d}$; ip); (2) Hpx group, hypoxic mother+0.9\% saline (1 mL/kg/d; ip); (3) Hpx-Spd group, hypoxic mother+SPD (5 mg/kg/d; ip); and (4) Hpx-SPDDFMO group, hypoxic mother+SPD ( $5 \mathrm{mg} / \mathrm{kg} / \mathrm{d}$; ip)+DFMO ( $5 \mathrm{mg} / \mathrm{kg} / \mathrm{d}$; ip). After birth, newborn pups from the above four groups were reared with their mothers and housed under room air conditions; after which, they were sacrificed and their hearts were extracted on postnatal day 7 for subsequent experiments.

2.3. Histological Analysis. For histological analyses, ventricular tissue was fixed in 4\% paraformaldehyde/phosphatebuffered saline (PBS) solution for 24-48 h. The tissue was subsequently dehydrated in a graded ethanol series, cleared in toluene, and embedded in paraffin. Five-micrometer paraffin sections were stained with hematoxylin-eosin ( $\mathrm{HE})$ and observed to assess overall cardiac morphology with an optical microscope (Eclipse E200; Nikon, Tokyo, Japan) [39]. Seven randomly chosen HE histological sections from each group of rats were analyzed by an expert pathologist.

\subsection{Evaluation of Cell Proliferation by Immunohistochemical} Analysis. For analysis of proliferation rates in neonatal hearts, immunohistochemical staining for MCM2 (minichromosome maintenance protein) was performed as previously described [40]. Briefly, after deparaffinization and rehydration of the tissues, inhibition of endogenous peroxidase was performed for $10 \mathrm{~min}$ with a solution of $0.3 \%$ hydrogen peroxide in PBS. Antigen retrieval was performed by heating for $5 \mathrm{~min}$ at $100^{\circ} \mathrm{C}$ in a citric acid buffer $(0.01 \mathrm{~mol} / \mathrm{L}$ in distilled water; $\mathrm{pH} 6.0)$. The tissues were incubated with $1 \%$ bovine serum albumin for $20 \mathrm{~min}$ and then incubated overnight with primary antibody against MCM2 (1:150, 105-1-AP, Proteintech, Wuhan, China). Between each subsequent incubation step, all slides were rinsed twice in PBS. The tissues were then incubated for $60 \mathrm{~min}$ with the HRP-labeled anti-rabbit IgG $(1: 200)$; after which, the slides were stained using a DAB Kit (AR1022, Boster, Wuhan, China) and counterstained with $0.1 \%$ hematoxylin for $5 \mathrm{~s}$, followed by rinsing for $10 \mathrm{~min}$ with tap water. All tissues were analyzed by light microscopy after they were dehydrated, sealed with neutral gums, and dried.

2.5. TdT-Mediated dUTP Nick End Labeling (TUNEL) Assay. The TUNEL assay was used to determine the number of apoptotic cells in the 7-day-old neonatal rat hearts. Heart tissues were treated following our previously described procedure [28] and labeled with TUNEL (Upstate Cell Signaling Solutions, Lake Placid, NY) according to the manufacturer's instructions [39]. Cells were counted and scored from five randomly selected areas on each slide, followed by quantitative analysis using Image-Pro Plus (Media Cybernetics, Silver Spring, MD).

2.6. Quantification of Fibrosis. Cardiac tissue sections from neonatal rats were collected as previously described and deparaffinized, rehydrated, and stained with Masson's trichrome following standard protocols [41]. Cardiac fibrosis was assessed by Masson's trichrome staining. Two nonadjacent cross sections per heart were used, and fibrosis was analyzed as a percentage of the fibrotic area to the total left ventricular myocardial area using the ImageJ software, v1.52 (NIH, Bethesda, MD). Perivascular fibrosis was excluded from the analysis.

2.7. Transmission Electron Microscopy (TEM). The cardiac apex tissues of neonatal rats in each group were dissected into approximately $1 \times 1 \times 1 \mathrm{~mm}$ pieces and randomly fixed in glutaraldehyde phosphoric acid buffer at $4^{\circ} \mathrm{C}$ for $2-4 \mathrm{~h}$. The specimens were then washed, dehydrated, embedded in resin, stained with uranyl acetate and lead nitrate, sectioned into 50-70 nm sections, and finally visualized with a TEM (H600; Hitachi, Tokyo, Japan).

2.8. Mitochondrial Morphological Analysis and Area Measurement. Morphological analysis of mitochondria was performed as previously described [42]. Briefly, two left ventricle tissue pieces from each rat were randomly chosen. Images were acquired at a magnification of $10,000 \mathrm{x}$ when an area containing longitudinal myofilaments surrounded by a mitochondrial network was observed. Individual mitochondria and myofilaments were delineated.

2.9. Mitochondrial Isolation. Mitochondria were isolated at $4^{\circ} \mathrm{C}$ by differential centrifugation as our previously described using a mitochondria isolation kit (Beyotime Biotechnology, Shanghai, China) [29]. Briefly, the neonatal rat hearts were collected for weighing, followed by washing with PBS. The hearts were cut into small pieces in a petri dish on ice; after which, 10 volumes of precooled PBS were added in the samples and incubated in an ice bath for $3 \mathrm{~min}$. The samples were then centrifuged at $600 \times g$ for $10-20 \mathrm{~s}$ to precipitate the tissue samples; the supernatant was discarded. After adding 8 volumes of precooled trypsin digest, the samples were incubated in an ice bath for $20 \mathrm{~min}$ and centrifuged again at $600 \times g$ for $10-20 \mathrm{~s}$ to precipitate the myocardial tissue; the supernatant was discarded. Eight volumes of mitochondrial separation reagent supplemented with phenylmethylsulfonyl fluoride (PMSF) before use were then added to the precipitate, homogenized 20-30 times in a homogenizer with an ice bath, and centrifuged for $5 \mathrm{~min}$ at $4^{\circ} \mathrm{C}$. The supernatant was transferred to another centrifuge tube and centrifuged at $11,000 \times g$ for $10 \mathrm{~min}$ at $4^{\circ} \mathrm{C}$; after which, the supernatant was carefully discarded, and the remaining precipitate contained the isolated mitochondria. The final cardiac mitochondrial pellet was then resuspended in isolation and homogenizing buffer, stored on ice, and used for experiments within $4 \mathrm{~h}$. Protein concentrations were determined by the BCA Protein Assay (Beyotime Biotechnology).

2.10. Measurement of Mitochondrial Oxygen Consumption. Mitochondrial oxygen consumption was measured using a Clark-type oxygen electrode (Hansatech Instruments, Norfolk, UK) at $25^{\circ} \mathrm{C}$ in mitochondrial respiration buffer (125 mM KCl, $5 \mathrm{mM} \mathrm{K} \mathrm{HPO}_{4}, 20 \mathrm{mM}$ HEPES, $2.5 \mathrm{mM}$ EGTA, and $1 \mathrm{mM} \mathrm{MgCl}_{2} \cdot 6 \mathrm{H}_{2} \mathrm{O} ; \mathrm{pH}$ 7.2) as previously 
described [29]. Pyruvate ( $5 \mathrm{mM})$ was used as a substrate for complex I-containing mitochondria at a final concentration of $500 \mu \mathrm{g}$ protein $/ \mathrm{mL}$. ADP-stimulated oxygen consumption (state 3 respiration) was measured in the presence of $200 \mu \mathrm{M}$ $\mathrm{ADP}$, and ADP-independent oxygen consumption (state 4 respiration) was monitored. The respiratory control ratio (RCR; state 3 divided by state 4 ) reflected oxygen consumption by phosphorylation (coupling).

2.11. Western Blot Analysis. To extract proteins from the 7-day-old neonatal rat heart tissues, frozen left ventricular cardiac tissues or isolated mitochondrial samples were homogenized in ice-cold RIPA lysis buffer (Beyotime Biotechnology) and $50 \mathrm{Lg} / \mathrm{mL}$ PMSF and then incubated on ice for $40 \mathrm{~min}$. The homogenate was centrifuged at 10,000 $\times \mathrm{g}$ for $15 \mathrm{~min}$ at $4^{\circ} \mathrm{C}$ to remove cellular debris and isolate the total protein extract. Cardiomyocytes were harvested and lysed in the same RIPA buffer containing PMSF. Protein concentrations were determined using a Bradford assay kit (Beyotime Biotechnology) as previously described [43]. Briefly, equal amounts of protein from different experimental groups were loaded and separated via 10\% SDS-PAGE; after which, the proteins were electrophoretically transferred to a $0.2 \mu \mathrm{m}$ pore polyvinylidene fluoride (PVDF) membrane (Merck Millipore, Burlington, MA). The membranes were blocked in TBS-T containing $5 \%(\mathrm{w} / \mathrm{v})$ skim milk at $37^{\circ} \mathrm{C}$ for $1 \mathrm{~h}$. Thereafter, the membranes were incubated overnight at $4^{\circ} \mathrm{C}$ with primary rabbit antibodies against GAPDH (1:2000; 10494-1-AP), VDAC $(1: 1000 ; 10866-1-A P)$, MFN2 ( $1: 1000 ; 12186-1$-AP), FIS1 (1:1000; 10956-1-AP), superoxide dismutase (SOD; $1: 2000 ; 24127-1$-AP), BAX (1:1000; 509599-2-Ig; all purchased from Proteintech, Chicago, IL), ODC (1:500; sc-33539), SSAT (1:1000; sc67159), DRP1 (1:1000; sc-271583), BCL2 (1:2000; sc-7382; all purchased from Santa Cruz Biotechnology, Dallas, TX), and PGC- $1 \alpha$ ( $1: 1000$; ab106814; Abcam, Cambridge, UK). Protein samples were fractionated using a PAGE Gel Rapid Preparation Kit (Beyotime Biotechnology) and transferred to PVDF membranes (Bio-Rad Laboratories, Hercules, CA). Samples were incubated overnight at $4^{\circ} \mathrm{C}$ with specific primary antibodies diluted in TBS-T. After washing, the samples were incubated with HRP-labeled goat anti-rabbit or HRP-labeled goat anti-mouse IgG secondary antibodies (Beyotime Biotechnology) at a dilution of $1: 6000$. Immunoreactive proteins were then developed using ultrasensitive ECL luminescent solution (Proteintech), quantified using a FluorChem Chemiluminescence Imaging System (ProteinSimple, San Jose, CA) via densitometry, and normalized to that of GAPDH. The final results were expressed as relative protein levels by normalizing the data to control values.

2.12. Quantitative Reverse Transcription PCR ( $q R T-P C R)$. Cardiac tissue samples were homogenized in the TRIzol reagent (Invitrogen, Carlsbad, CA), and total RNA was isolated according to the manufacturer's instructions. mRNA was reverse transcribed with 2X SYBR Green qPCR Master Mix [44]. qPCR was performed on a LightCycler 96 RealTime PCR System (Roche, Basel, Switzerland) according to the manufacturer's instructions. Gene-specific primers were designed using the Primer Express software (Thermo Fisher Scientific, Waltham, MA) and synthesized by Sangon Biotechnology (Shanghai, China) as follows, Mfn2, forward: $5^{\prime}$-CTC AGG AGC AGC GGG TTT ATT GTC T-3' and reverse: $5^{\prime}$-TGT CGA GGG ACC AGC ATG TCT ATC T-3'; Fis1, forward: $5^{\prime}$-GTG CCT GGT TCG AAG CAA ATA C- $3^{\prime}$ and reverse: $5^{\prime}$-CAT AAT CCC GCT GCT CCT CTT-3'; Drp1, forward: $5^{\prime}$-CGT AGT GGG AAC TCA GAG CA-3' $3^{\prime}$ and reverse: $5^{\prime}$-TGG ACC AGC TGC AGA ATA AG- $3^{\prime}$; and PGC- $1 \alpha$, forward: $5^{\prime}$-GTG CAG CCA AGA CTC TGT ATG G- $3^{\prime}$ and reverse: $5^{\prime}$-GTC CAG GTC ATT CAC ATC AAG TTC-3'. All primers and PCR conditions were optimized to PCR efficiencies between $90 \%$ and $110 \%$ and a correlation coefficient of $\geq 0.990$ using a cDNA dilution series. All samples were analyzed in triplicate. Relative mRNA levels were quantified using a standard curve and normalized to GAPDH levels. Relative gene expression was analyzed using the $2^{-\Delta \Delta C T}$ method.

2.13. Hypoxic Cardiomyocyte Model. Neonatal rat hearts were enzymatically dissociated into a single cell suspension as previously reported [40]. Briefly, the hearts were rapidly excised and rinsed in PBS solution. The hearts were minced and incubated in $0.25 \%$ trypsin and $0.02 \%$ EDTA (Beyotime Biotechnology) at $37^{\circ} \mathrm{C}$ for $8 \mathrm{~min}$. The obtained suspensions were then incubated for 2-3 min to precipitate undissociated tissue fragments. The supernatant was then centrifuged at $800 \times \mathrm{g}$ for $10 \mathrm{~min}$. The precipitate was transferred to DMEM supplemented with $10 \%$ fetal calf serum (Biolot, Saint Petersburg, Russia), $50 \mathrm{U} / \mathrm{mL}$ penicillin, and $50 \mu \mathrm{g} / \mathrm{mL}$ streptomycin, followed by $1 \mathrm{~h}$ preincubation in a glass petri dish to eliminate nonmyocytic cells. The cells were plated at an initial density of $1 \times 10^{5}$ cells $/ \mathrm{mL}$ and cultured for $72 \mathrm{~h}$ in a $\mathrm{CO}_{2}$ incubator at $37^{\circ} \mathrm{C}$ and $5 \% \mathrm{CO}_{2}$. The cells were then placed in a glass hypoxic chamber (BioSpherix OxyCycler C42; Redfield, NY), which was opened with an exhaust valve and filled with nitrogen for $8 \mathrm{~min}$; no residual oxygen was found in the warehouse. The cultured neonatal rat cardiomyocytes (NRMCs) were then randomly divided into four groups: (1) control group (Con), cells cultured under normal incubation conditions; (2) hypoxia ( $\mathrm{Hpx}$ ) group, cells placed in a hypoxic chamber for $24 \mathrm{~h}$ and then cultured normally for $24 \mathrm{~h}$; (3) Hpx-Spd group, cells placed in a hypoxic chamber and incubated with $10 \mu \mathrm{mol} / \mathrm{L}$ SPD for $24 \mathrm{~h}$; (4) and HpxSpd-DFMO group, cells placed in a hypoxic chamber and incubated with $10 \mu \mathrm{mol} / \mathrm{LSPD}+2 \mathrm{mmol} / \mathrm{L}$ DFMO for $24 \mathrm{~h}$.

2.14. Mitochondrial Superoxide Production. MitoSOX Red is a mitochondrial superoxide indicator dye used to detect superoxide production in the mitochondria of living cells and determine the extent of oxidative stress [45]. The MitoSOX Red reagent is oxidized by superoxide in the mitochondria, leading to strong red fluorescence. NRCMs in each group were washed with PBS and incubated with $5 \mu \mathrm{M}$ MitoSOX Red according to the manufacturer's instructions. After a $10 \mathrm{~min}$ incubation period in the dark, the cells were washed again with PBS, followed by measuring MitoSOX 
Red fluorescence using excitation and emission wavelengths of 510 and $580 \mathrm{~nm}$, respectively. Cardiomyocytes in each group were randomly imaged, and their total fluorescence was recorded and assayed.

2.15. Measuring $\Delta \Psi m$. Isolated and cultured NRCMs were incubated with tetramethylrhodamine ethyl ester (JC-1;1 $1 \mu \mathrm{g} / \mathrm{mL}$ ) for $20 \mathrm{~min}$ in the dark in a cell incubator according to the manufacturer's instructions (Beyotime Biotechnology). NRCMs were washed twice with PBS and then immediately observed by fluorescence microscopy (BX51M; Olympus, Tokyo, Japan). Cardiomyocytes in each group were randomly imaged. Changes in $\Delta \Psi \mathrm{m}$ were assessed by comparing the optical density ratios at 590$600 \mathrm{~nm}$ (red) to $527-534 \mathrm{~nm}$ (green) [46]. The ratio of intensity of the JC-1 aggregate (red) to that of the monomer (green) was calculated.

2.16. Mitochondrial Imaging. For mitochondrial staining, NRCMs were incubated in 200 nM MitoTracker ${ }^{\circledR}$ Green FM (dissolved in DMSO; Invitrogen) and preheated in culture medium at $37^{\circ} \mathrm{C}$ for $30 \mathrm{~min}$ as previously described $[47,48]$. Briefly, the cells were washed and fixed with $4 \%$ paraformaldehyde for $10 \mathrm{~min}$ and then washed again and observed under a fluorescence microscope. An increase in the fragmentation pattern and mitochondrial number together with a decrease in mean mitochondrial volume was considered the criteria for mitochondrial fission. Within cells, two to three regions of interest (ROI) of an equal area were defined, and mitochondrial counts and volume were measured for each ROI. Each experiment was performed four times and 16-25 cells/condition were quantified.

2.17. Statistical Analysis. Data are expressed as the mean \pm SEM. One-way analysis of variance was used for the statistical evaluation of differences among groups. Unpaired data were analyzed using Student's $t$-test where appropriate. All statistical analyses were performed using SPSS 13.1 (IBM, Armonk, NY) and GraphPad Prism 8 software (GraphPad Software Inc., La Jolla, CA). $P$ values $<0.05$ were considered statistically significant.

\section{Results}

3.1. PA Metabolism in the Neonatal Heart. We analyzed the effect of IUH on myocardial PA metabolism in the neonatal rats by determining the protein levels of the key PA synthesis enzyme ODC and the key PA catabolic enzyme SSAT in 7-day-old rats exposed to IUH (Figure 1). The results showed that ODC protein expression was decreased and SSAT protein expression was increased compared with those of the control group. These findings indicate that IUH altered myocardial PA metabolism in the neonatal offspring, leading to decreased PA synthesis and increased decomposition.

3.2. Offspring and Heart Characteristics. Body weight (BW) and heart weight (HW) of 7-day-old rats was measured and the $\mathrm{HW}$ to $\mathrm{BW}$ ratio $(\mathrm{HW} / \mathrm{BW})$ was calculated (Figure 2(c)). IUH led to decreased BW and $\mathrm{HW}$ $(P<0.05)$, while $\mathrm{HW} / \mathrm{BW}$ increased in the offspring heart
$(P<0.05)$. Exogenous SPD administration increased BW and $\mathrm{HW}$ of neonatal rats $(P<0.05)$ and significantly decreased HW/BW compared with that of the hypoxia group $(P<0.05)$. Meanwhile, the PA synthesis inhibitor DFMO abolished the effects induced by SPD $(P<0.05)$. These results demonstrate that chronic hypoxia during pregnancy resulted in offspring growth retardation and that SPD treatment during pregnancy inhibited changes caused by IUH, indicating that PAs can prevent the growth retardation induced by IUH.

3.3. Effects of SPD on Myocardial Morphological Structure, Cell Proliferation, Apoptosis, and Fibrosis in Offspring Exposed to IUH. Myocardial morphological structure, cell proliferation, apoptosis, and myocardial fibrosis were analyzed in the rat hearts of 7-day-old offspring. Compared with normal hearts, neonatal offspring hearts exposed to IUH showed loosely arranged cardiac muscle fibers and increased interstitial distances as evaluated by HE staining. However, hearts in the IUH group treated with SPD maintained good myocardial histological structures compared with those of control hearts (Figure 3(a)). We also examined the number of binucleated cardiomyocytes and expression of the nuclear proliferation protein MCM2 as they reflect the degree of cardiomyocyte maturation and differentiation. Analysis of the HE-stained samples showed a higher proportion of binucleated cardiomyocytes in rat hearts exposed to IUH compared with control hearts $(P<0.05)$. Meanwhile, the proportion of binucleated cardiomyocytes was significantly reduced in the hearts of offspring with SPD-treated mothers compared with heart suffered from IUH $(P<0.05)$; DFMO attenuated the effects of SPD $(P<0.05$; Figures $3(\mathrm{a})$ and $3(d))$. MCM2 expression in the IUH group was also significantly lower than that in the control group $(P<0.05)$ and was strongly expressed in the SPD group; DFMO again abolished the effect of SPD on MCM2 expression (Figures 3(b) and 3(e)). These results indicate that the PA SPD can promote myocardial proliferation in neonatal offspring exposed to IUH.

We further counted TUNEL-positive nuclei to determine the cell apoptosis ratio (Figures 3(c) and 3(f)) and found that the number of TUNEL-positive cardiomyocytes was substantially higher in neonatal rat hearts exposed to IUH than in control rat hearts $(P<0.05)$. In contrast, the ratio of TUNEL-positive cells in SPD-treated rats $(P<0.05)$ was lower than that in untreated hypoxic rats. As mitochondrial damage can induce the mitochondrial pathway of apoptosis, we analyzed the expression levels of proapoptotic protein BAX and antiapoptotic protein BCL2 in neonatal hearts by immunoblotting (Figures 3(g) and 3(h)) and found that the BAX/BCL2 protein expression ratio was significantly higher in the myocardium of the IUH group than of the control group $(P<0.05)$. However, in the myocardium of offspring exposed to IUH and administered with SPD, the BAX/BCL2 ratio was decreased compared with the IUH group $(P<0.05)$; expectedly, DFMO abolished the effects of SPD $(P<0.05)$. These results indicate that the PA SPD can inhibit cardiomyocyte apoptosis in the myocardium of neonatal offspring exposed to IUH. 


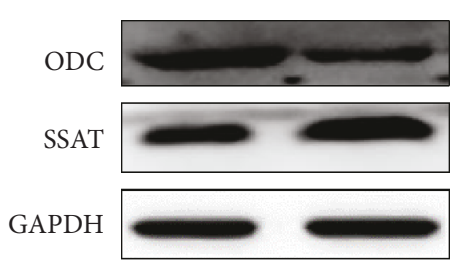

(a)

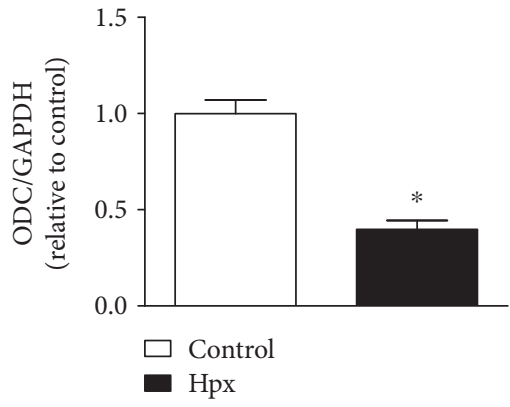

(b)

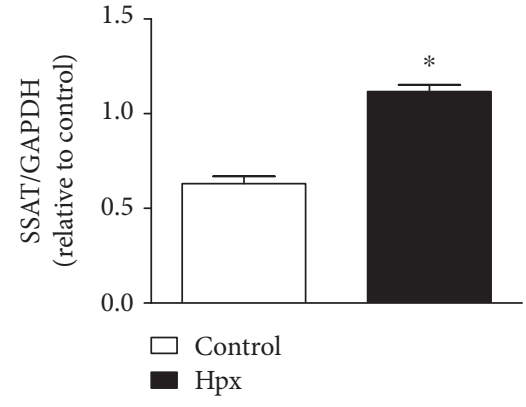

(c)

FIgure 1: Polyamine metabolism in the neonatal rat hearts. Protein levels of ornithine decarboxylase (ODC) and spermidine/spermine acetyltransferase (SSAT) in cardiac tissue collected from neonatal offspring were determined by western blotting. Data are shown as the mean \pm SEM; $n=4$ per group. ${ }^{*} P<0.05$ versus control. Hpx: hypoxia.

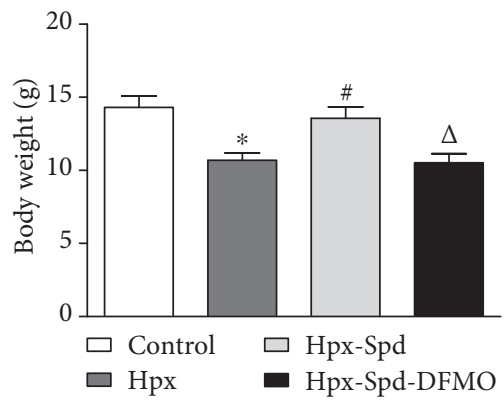

(a)

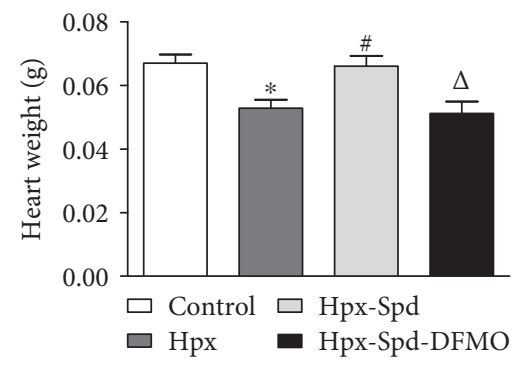

(b)

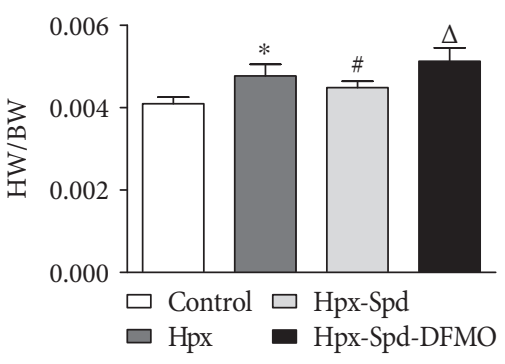

(c)

FIGURE 2: Evaluation of neonatal offspring and heart characteristics. (a) Body weight (BW), (b) heart weight (HW), and (c) BW/HW ratio of neonatal rats. Data are shown as the mean \pm SEM; $n=8$ per group. ${ }^{*} P<0.05$ versus control, ${ }^{\#} P<0.05$ versus the Hpx group, and ${ }^{\triangle} P<0.05$ versus the Hpx-Spd group. Hpx: hypoxia; Hpx-Spd: hypoxia and SPD treatment; Hpx-Spd-DFMO: hypoxia and SPD+DFMO treatment.

Next, we evaluated myocardial fibrosis using a collagen area assay (Figures 3(i) and $3(\mathrm{j})$ ) and observed increased myocardial collagen deposition in neonatal rat hearts exposed to IUH, which was higher than that of the control group $(P<0.05)$. Conversely, the area of fibrosis was decreased to a greater extent after SPD treatment compared with the IUH group $(P<0.05)$. Moreover, compared with the SPD treatment group, the Hpx-Spd-DFMO group showed a significantly enhanced fibrotic area $(P<0.05)$. Overall, our results suggest that SPD ameliorates the decreased proliferation as well as the increased apoptosis and collagen deposition in the offspring heart exposed to IUH.

3.4. Effects of SPD on Mitochondrial Structure and Function as well as SOD/ROS Production and $\triangle \Psi m$ in Offspring and Primary Cardiomyocytes Exposed to Hypoxia. Changes in the myocardium and myocardial mitochondrial ultrastructure in neonatal rats were analyzed by TEM (Figures 4(a) and 4(b)). Mitochondrial content (\% of the mitochondrial area compared with the whole-cell area) and mitochondrial area were quantified using ImageJ (Figures 4(c) and 4(d)). In normal rats, well-developed myocardial mitochondria with preserved membranes and cristae were observed; in contrast, decreased mitochondrial matrix density, disorganized mitochondrial cristae, and swollen, small, and irregular mitochondria were observed in some cardiomyocytes of hypoxic rats. However, in offspring hearts exposed to IUH and SPD treatment, the mitochondria were more tightly packed between the myofibrils and exhibited intact outer and inner membranes with distinct cristae. Compared with those of the IUH group, the proportion of mitochondria in myocardial cells and the mitochondrial area increased following SPD treatment; DFMO inhibited these SPD-induced effects.

We next measured mitochondrial respiratory function, including state 3 and 4 respiratory rates and the RCR (Figures $4(\mathrm{e})-4(\mathrm{~g}))$, using pyruvate/malate as substrates. We found that state 3 and 4 respiratory rates as well as the RCR were significantly decreased in the IUH group compared with the control group $(P<0.05)$. Interestingly, state 3 and 4 respiratory rates and the RCR were restored by SPD treatment $(P<0.05)$; conversely, these SPD effects were significantly inhibited in the DFMO-treated group. These findings suggest that SPD can inhibit the decrease in mitochondrial respiratory function of neonatal rat hearts exposed to IUH.

We further determined the expression levels of the antioxidative enzyme SOD in the offspring myocardium (Figures 4(h) and 4(i)) and found that SOD protein expression in the myocardium of the IUH group was significantly decreased compared with that of the control group 
(a)

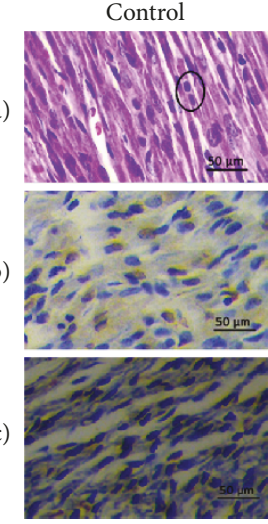

Hpx
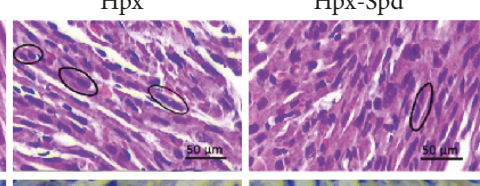

Hpx-Spd-DFMO

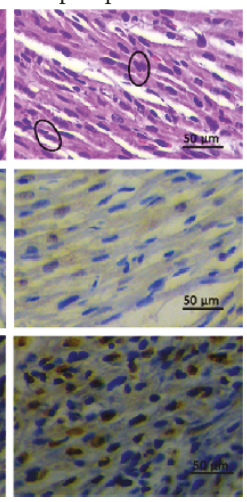

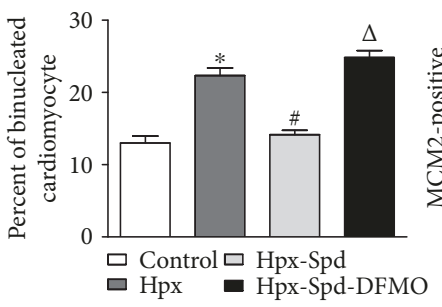

(d)

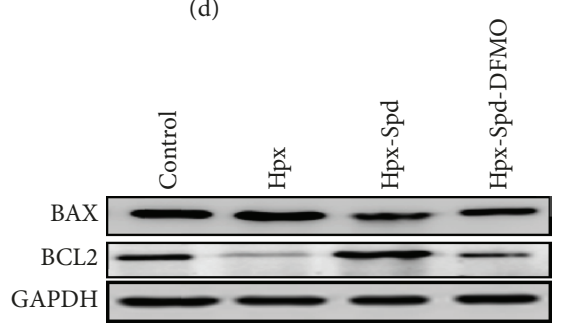

(g)

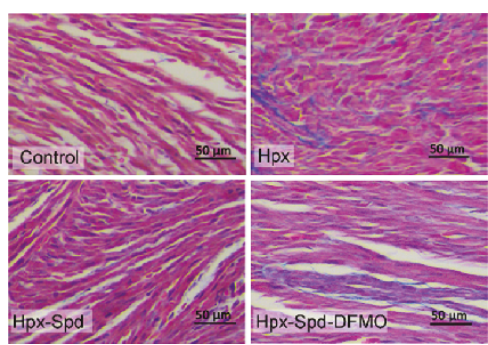

(i)

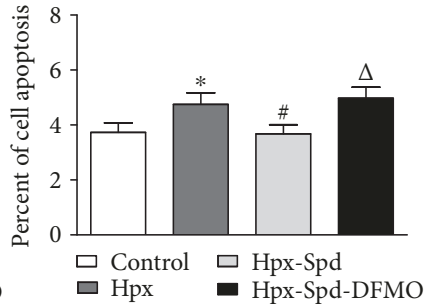

(f)

(e)

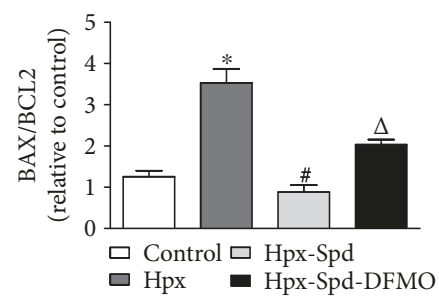

(h)

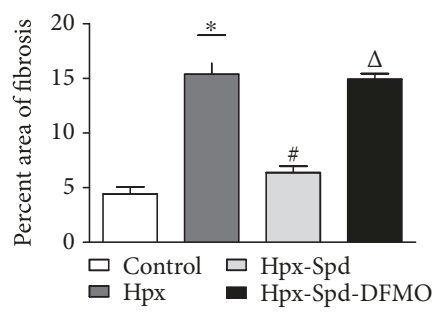

(j)

FIGURE 3: Observation of myocardial morphological structure, cell proliferation, apoptosis, and fibrosis in offspring rats. (a) Representative left ventricle sections stained by $\mathrm{HE}$ in the normal (control), hypoxia (Hpx), SPD-treated (Hpx-Spd), and SPD+polyamine synthesis inhibitor- (Hpx-Spd-DMFO-) treated groups. (b) Representative immunohistochemical staining for protein expression and localization of MCM2-positive cardiomyocytes. (c) Brown-stained nuclei indicate TUNEL-positive cells. (d) Evaluation of the percentage of binucleated cardiomyocytes $(n=6)$. (e) Evaluation of the percentage of MCM2-positive cells $(n=10)$. (f) The percentage of TUNEL-positive nuclei in different groups $(n=8)$. (g) BAX and BCL2 protein expression detected by western blotting. (h) Quantification of the BAX and BCL2 protein level ratio $(n=4)$. (i) Representative Masson's trichrome staining in ventricle sections in each group. (j) Evaluation of interstitial fibrotic areas in ventricle sections in each group $(n=8)$. Data are shown as the mean \pm SEM. ${ }^{*} P<0.05$ versus control, ${ }^{\#} P<0.05$ versus the Hpx group, and ${ }^{\triangle} P<0.05$ versus the Hpx-Spd group.

$(P<0.05)$. In the presence of SPD treatment, however, SOD levels in the IUH offspring myocardium were found increased $(P<0.05)$. DFMO once again abolished the effects of SPD $(P<0.05)$. The results indicate that in utero PA treatment can reverse the decrease in offspring myocardial antioxidants induced by IUH exposure.

Next, we examined mitochondrial ROS levels in vitro using MitoSOX Red (Figures 4(j) and 4(k)) and found that 

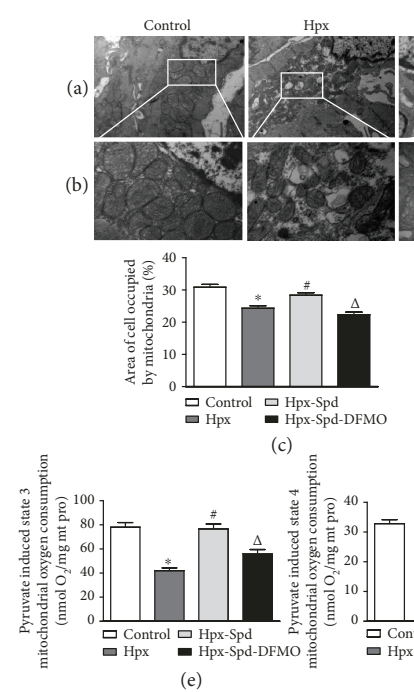

(c)
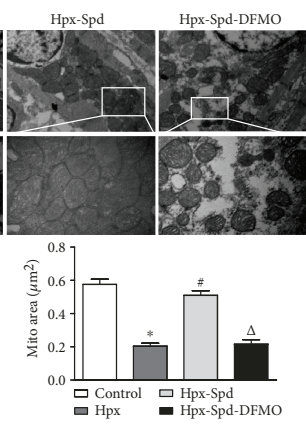

(d)
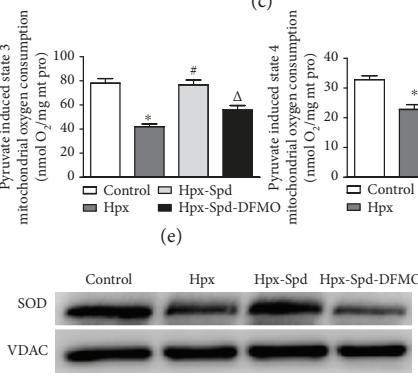

(h)

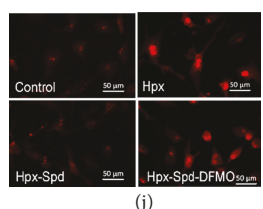

(i)

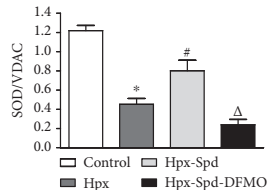

(g)
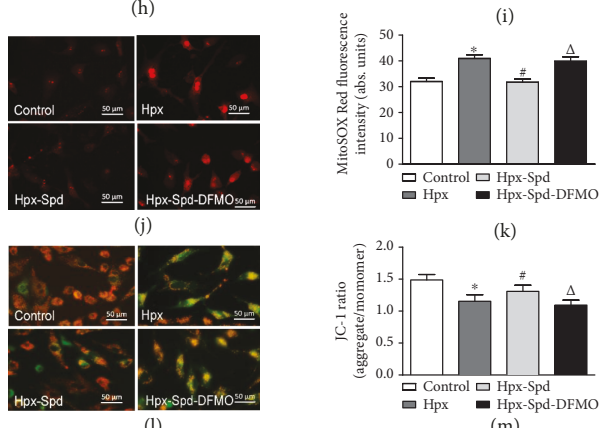

(k)

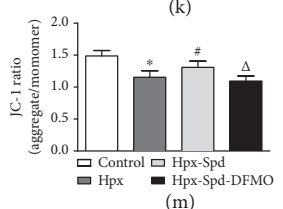

FIGURE 4: Effects of SPD on mitochondrial quantity and quality, mitochondrial function, and MnSOD expression in the myocardium of neonatal offspring and on mitochondrial ROS production and membrane potential in primary cardiomyocytes (NRCMs). (a) TEM of myocardial ultrastructure of normal (control), hypoxia (Hpx), SPD-treated (Hpx-Spd), and SPD+polyamine synthesis inhibitor- (HpxSpd-DMFO-) treated groups (magnification, 10,000x). (b) Representative TEM images showing ultrastructural changes in the myocardial mitochondria of each group (magnification, 30,000x). (c, d) Quantification of the area of cells occupied by mitochondria (\%) and the quantitative mitochondrial area $(n=10)$. (e-g) Mitochondrial function was evaluated based on mitochondrial state 3 (e) and state 4 (f) oxygen consumption and the respiratory control rate (RCR) (g) in cardiac mitochondria isolated from 7-day-old offspring hearts $(n=10)$. (h) The expression ratio of SOD proteins in isolated mitochondria detected by western blotting. (i) Quantification of the protein levels $(n=4)$. (j) Detection of superoxide production in myocardial mitochondria of NRCMs by the MitoSOX Red fluorescent probe. (k) Statistical quantification of the average fluorescence intensity of MitoSOX Red $(n=6)$. (l) Detection of mitochondrial membrane potential $(\triangle \Psi \mathrm{m})$ of NRCMs by the JC-1 fluorescent probe. $(\mathrm{m})$ Statistical analysis of the ratio of red to green fluorescence $(n=6)$. Data are shown as the mean \pm SEM. ${ }^{*} P<0.05$ versus control, ${ }^{\#} P<0.05$ versus the Hpx group, and ${ }^{\triangle} P<0.05$ versus the Hpx-Spd group.

ROS production increased after cardiomyocytes were exposed to hypoxia for $24 \mathrm{~h}$; conversely, SPD inhibited the hypoxia-induced ROS production, which was relieved when cardiomyocytes were also treated with DFMO. Furthermore, we measured $\Delta \Psi \mathrm{m}$ in neonatal rat cardiomyocytes to evaluate the effects of SPD on $\triangle \Psi \mathrm{m}$ depolarization in hypoxic cardiomyocytes (Figures 4(l) and 4(m)). The JC-1 fluorescent probe was used to label cardiomyocytes and monitor changes in $\Delta \Psi \mathrm{m}$. Red fluorescence indicated that the mitochondrial membrane was intact, whereas green fluorescence indicated that it was destroyed and $\Delta \Psi \mathrm{m}$ was decreased; the red and green fluorescence ratios represent $\Delta \Psi \mathrm{m}$. We found that hypoxia exposure decreased $\Delta \Psi \mathrm{m}$, whereas SPD inhibited the hypoxia-induced decrease in $\Delta \Psi \mathrm{m}$.

\subsection{Effects of SPD on Mitochondrial Biogenesis and Fission/} Fusion Dynamics in Offspring and Primary Cardiomyocytes Exposed to Hypoxia. Mitochondrial morphology is regulated by a family of mitochondrial fusion and fission proteins; therefore, we investigated expression changes in the mitochondrial fusion protein MFN2, the fission proteins FIS1 and DRP1, and the mitochondrial biogenesis key regulatory protein PGC- $1 \alpha$ at the mRNA and protein levels (Figures 5(a)-5(c)). We found that the mRNA levels of 


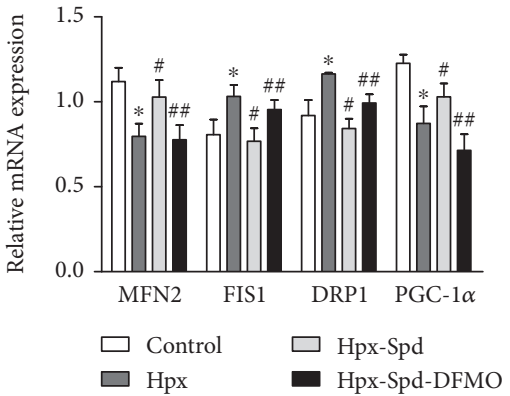

(a)

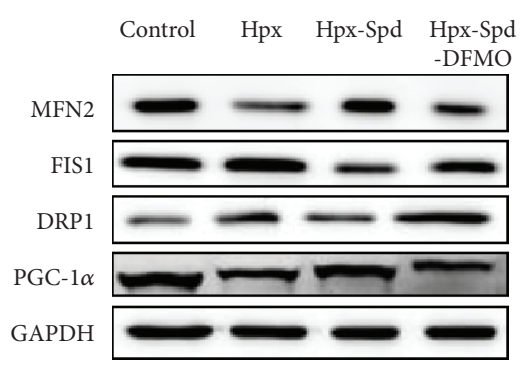

(b)

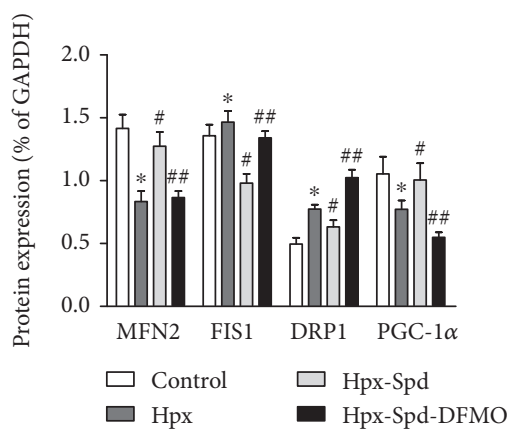

(c)

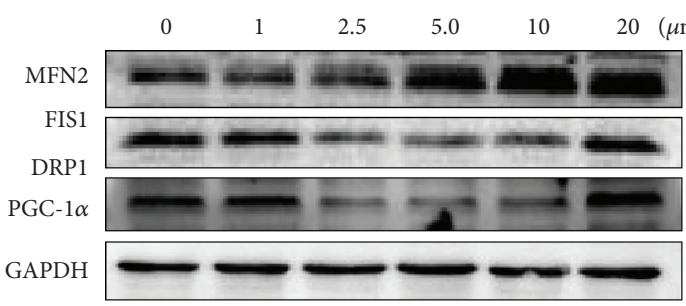

(d)

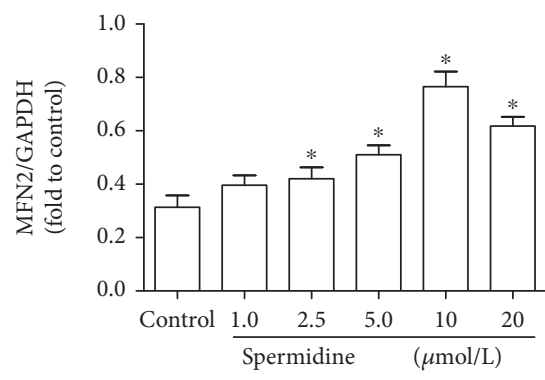

(e)

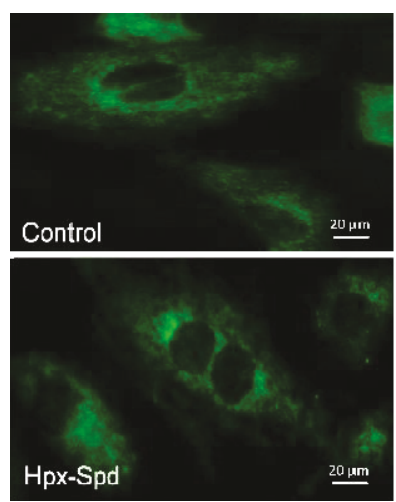

(h)

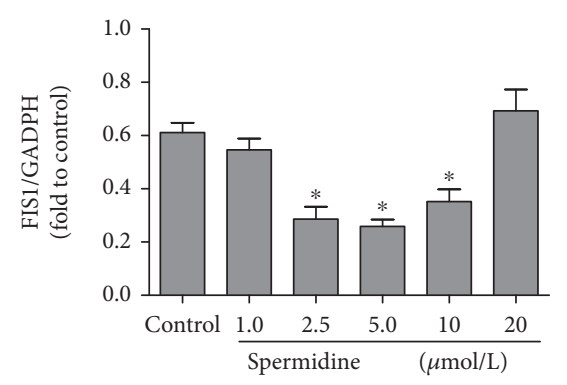

(f)

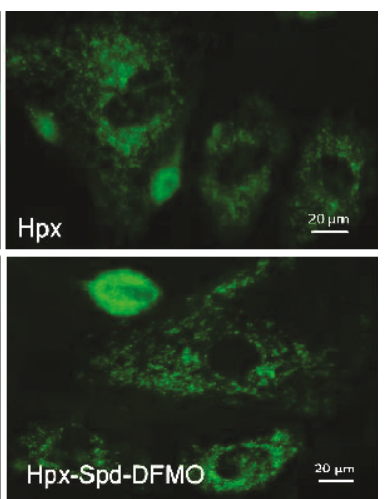

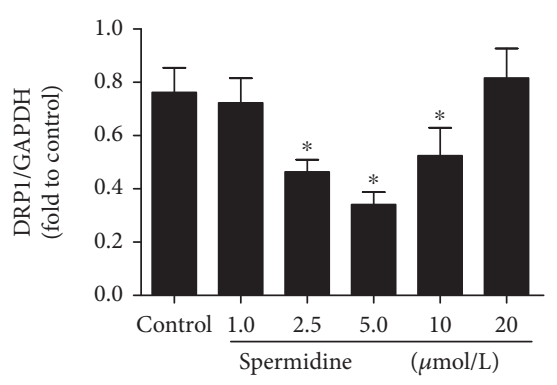

(g)

FIGURE 5: Effects of SPD on mitochondrial biogenesis and fission/fusion dynamics in the offspring myocardium and NRCMs exposed to hypoxia. (a) mRNA expression levels of myocardial MFN2, FIS1, DRP1, and PGC-1 $\alpha$ were determined by qRT-PCR; GAPDH was used as an internal control $(n=6)$. (b) Protein levels of MFN2, FIS1, DRP1, and PGC-1 $\alpha$ in the offspring myocardium collected from the control, Hpx, Hpx-Spd, and Hpx-Spd-DFMO groups were assessed by western blotting. (c) Quantification of MFN2, FIS1, DRP1, and PGC-1 $\alpha$ protein levels normalized to GAPDH in each group $(n=4)$. Data are shown as the mean \pm SEM. ${ }^{*} P<0.05$ versus control, ${ }^{\#} P<0.05$ versus the Hpx group, and ${ }^{\triangle} P<0.05$ versus the Hpx-Spd group. (d) Western blotting showed a dose-dependent effect of SPD (0, 2.5, 5, 10, and $20 \mu \mathrm{mol}$ ) on the protein levels of MFN2, FIS1, and DRP1 in NRCMs after exposure to hypoxia. Quantitative analysis of (e) MFN2, (f) FIS1, and (g) DRP1 protein levels normalized to that of GAPDH. Data are shown as the mean \pm SEM. ${ }^{*} P<0.05$ versus the control group $(0 \mu \mathrm{mol} / \mathrm{L}$ SPD treatment; $n=6)$. (h) Detection of fused and fragmented mitochondria in NRCMs by MitoTracker Green staining (magnification, 400x). (i) The percentage of fragmented mitochondria. $n=6$ for each group. Data are shown as the mean \pm SEM. ${ }^{*} P<0.05$ versus the control group, ${ }^{\#} P<0.05$ versus the Hpx group, and ${ }^{\triangle} P<0.05$ versus the Hpx-Spd group. 
MFN2 and PGC- $1 \alpha$ were significantly decreased, whereas those of FIS1 and DRP1 were increased in the hypoxia group compared with the control group $(P<0.05)$. Administration of SPD increased MFN2 and PGC- $1 \alpha$ mRNA levels and decreased FIS1 and DRP1 levels compared with that of the hypoxia group $(P<0.05)$; conversely, DMFO treatment abolished the effects induced by SPD $(P<0.05$; Figure 5(a)). Similarly, the changes in MFN2, FIS1, DRP1, and PGC- $1 \alpha$ levels at the protein level were consistent with the trend observed for mRNA (Figures 5(b) and 5(c)).

We further examined the effects of different concentrations of SPD on the expression of mitochondrial fusion and fission proteins in primary cultured NRCMs. We found that SPD increased Mfn2 and decreased Fis1 and Drp1 expression in a dose-dependent manner (Figure 5(d)). The optimal concentration of SPD for promoting mitochondrial fusion and reducing fission was $10 \mu \mathrm{mol}$ and $5 \mu \mathrm{mol}$, respectively (Figures $5(\mathrm{e})-5(\mathrm{~g})$ ). Therefore, $5 \mu \mathrm{mol} \mathrm{SPD}$ was used to examine the effect of SPD on mitochondrial fusion and fission in hypoxic NRMCs using the MitoTracker ${ }^{\circledR}$ Green fluorescence probe. The results demonstrated that hypoxia disrupted the microtubule networks, whereas SPD treatment significantly inhibited the hypoxia-induced mitochondrial fragmentation in NRCMs (Figures 5(h) and 5(i)).

\section{Discussion}

To our knowledge, we demonstrated for the first time that maternal hypoxic exposure during the late stages of fetal development results in decreased anabolism and increased catabolism of PAs in the cardiac tissue of newborn rats. Physiological levels of PAs are critical for cell function. PAs are ROS scavengers, protecting DNA, proteins, and lipids from oxidative damage [48]. More importantly, PAs exert positive effects during embryonic and fetal development, implantation, embryonic diapause, placentation, and angiogenesis [49]. Evidence shows that ODC activity is rapidly increased in the rat uterus after embryo implantation, which brings about the accumulation of PAs in the uterus, placenta, and fetus [50]. In our present study, ODC expression decreased while SSAT expression increased in the cardiac tissues of newborn rats exposed to IUH, which led to reduced levels of protective PAs that was coupled with an increase in the by-product $\mathrm{H}_{2} \mathrm{O}_{2}$ and production of 3-aminoacetaldehyde due to PA catabolism, which was sufficient to cause oxidative damage.

Experimental evidence from human and animal models indicates that IUH-induced placental insufficiency exposes the developing fetus to oxidative and hemodynamic stress, which in turn directly and indirectly impacts the developing cardiovascular system [51]. Thus, we speculated that metabolic disturbance in PAs leads to decreased PAs in the cardiac tissue of newborn offspring, which can be traced back to the decrease in PA levels in placental tissue and the fetus during fetal development and maternal hypoxia. Several studies support this notion; for example, Basystyi [52] revealed that PA concentrations in erythrocytes in the blood of pregnant women with IUGR are drastically lower than those of pregnant women with a normal physiological pregnancy and that the progression in PA imbalance depended on the severity of fetal growth retardation in pregnant women [52]. Another study reported that maternal blood PAs are possibly of fetal origin and that a modified pattern in maternal PAs may indicate insufficient fetal growth [53]. A recent study also suggested that placental PA metabolism differs depending on fetal growth restriction and that maternal serum levels of PA metabolites are strongly associated with obstetrical syndromes [54]. Nonetheless, more evidence is required to explore the role of PAs in the interactions between the fetus and mothers and in fetal and neonatal heart development.

In the present study, we also found that maternal hypoxia exposure led the newborn offspring to exhibit significantly decreased BW and HW and elevated apoptosis and fibrosis and caused cardiomyocytes to exit from the cell cycle ahead of schedule, as indicated by an increase in the number of binucleated cardiomyocytes and MCM2-positive nuclei. This indicates that the IUH resulted in impaired postnatal cardiac growth plasticity. Similarly, several studies also demonstrated programming effects of prenatal hypoxia in the mammalian hearts of IUH rodent model offspring $[43,55,56]$. It is important to mention that a number of studies reported that maternal dietary supplementation with Arg, a major substrate of PA production, increases BW and decreases perinatal mortality in rat, ewe, and rabbit models exposed to IUGR during the gestation period [22, 57, 58]. Moreover, supplementation with Arg was sufficient to increase SPD concentrations in colonic tissue and blood [59, 60]. Of note, a previous study revealed that SPD, but not SP, is essential for early development, organogenesis, and color pattern formation and that maternal supplementation of SPD synthase in zebrafish is sufficient to rescue early developmental defects [34]. A similar earlier study also suggested that uterine and fetal SPD concentrations show sustained elevation during the early stage of gestation in mice, whereas the SP concentration remains relatively stable. Furthermore, they demonstrated the essential role of SPD in murine embryogenesis using the highly selective ODC inhibitor DFMO, which markedly reduced ODC activity and PU and SPD concentrations in uterine tissue. Administration of DFMO results in fetal developmental failure between days 9 and 12 of gestation. Mice, rat, and rabbit were treated with DFMO during the late stage of gestation showing a significant difference from controls in average fetal numbers and weight $[61,62]$. DFMO results in depletion of $\mathrm{Pu}$ and SPD; however, only incomplete depletion of SP occurs [63].

The broad role of SPD in disease and health has recently received increased attention [31,32]. Therefore, we evaluated the effect of PA SPD administration during late pregnancy stages on the newborn myocardium by employing a rat model of prenatal hypoxia. Our findings demonstrated that SPD supplementation to the hypoxic mother rescued neonatal BW and HW, decreased the ratio of HW to BW, and maintained good myocardial histological structures in newborn rats. SPD treatment also decreased the ratio of BAX to BCL2 expression and percentage of TUNEL-positive nuclei as well as inhibited myocardial fibrosis (collagen deposition) within the myocardium of IUH-exposed neonates, reversing the effects seen under hypoxia exposure. Conversely, DFMO 
given to the mother during the late stage of gestation abolished the SPD-mediated protection for the neonatal heart, and this may be due to the deep depletion of cardiac polyamine pool especially SPD by DFMO on one hand; on the other hand, DFMO given to the mother suffering from IUH could cause more serious damage to the placenta and developing fetus than IUH treatment, both of which together contributed to the failure of the protection induced by exogenous SPD. These results indicate that SPD treatment in utero is beneficial for preventing newborn cardiac impairment induced by maternal hypoxia.

Mitochondria are a major source of ROS during prenatal hypoxia, which leads to placental and neonatal oxidative stress and fetal programming of CVD [5]. Neary et al. [47] reported that growth restriction following uteroplacental insufficiency programs reduced cardiac gene expression of the key mitochondrial antioxidant enzyme MnSOD in offspring in later postnatal life [47]. A recent study also revealed that prenatal hypoxia reduced the expression of subunits of the electron transport chain mitochondrial proteins, thereby reducing the mitochondrial respiration rate in the offspring hearts [9]. In addition, other studies showed that maternal exposure to adverse factors (placental insufficiency or high altitudes) during pregnancy will program fetal mitochondria [64]. Similar to these studies, our findings demonstrated that the hearts of newborn offspring exposed to maternal hypoxia showed a reduced mitochondrial area and proportion of mitochondria within cardiomyocytes, decreased mitochondrial respiratory function, and lower antioxidant SOD levels. These results indicate that fetal oxidative stress and mitochondrial dysfunction induced by IUH may persist until after birth, leading to cardiac impairment in the newborn offspring. As mitochondria provide energy for proper cell function, a reduction in number or functional alterations are likely to be detrimental for cells, particularly for cardiomyocytes with high energy requirements [65]; thus, maternal hypoxia is especially detrimental for the developing neonatal heart.

Previous studies have shown that prenatal antioxidant supplementation with resveratrol [66], ascorbic acid [67], or melatonin [68] as well as physical exercise [69] can rescue cardiovascular dysfunction in the fetus during hypoxia and prevent the programming of CVD in adults. In the present study, SPD treatment during hypoxic pregnancies promoted morphological and respiratory function recovery of myocardium mitochondria and elevated SOD levels in the hearts of newborn offspring compared with those exposed to prenatal hypoxic conditions. SPD almost completely blocked the increase in mitochondrial ROS levels and membrane potential depolarization in NRCMs under hypoxic conditions. Conversely, DFMO treatment abolished the SPD-induced effects in vivo and in vitro. Thus, our findings provide strong evidence that maternal SPD treatment can protect the fetus and newborns from hypoxia-induced cardiac oxidative stress and mitochondria injury during hypoxiacomplicated pregnancies.

Cardiac mitochondria are dynamically regulated to generate and consume enormous amounts of ATP to support the constant pumping action of the heart. Excessive mitochondrial fission was shown to inhibit mitochondrial respiratory function and increase ROS production [70], whereas the inhibition of mitochondrial fusion can result in loss of mitochondrial DNA [71]. Our present study showed that prenatal hypoxia led to a decrease in mitochondrial biogenesis and an imbalance in mitochondrial dynamics, which was reversed by prenatal SPD treatment. After birth, the heart commences its aerobic metabolism and cardiac mitochondrial biogenesis is increase [72]. Mitochondrial biogenesis regulates mitochondrial dynamics via effects on fusion and fission for mitochondrial maturation in postnatal hearts $[73,74]$. Recent evidence from animal experiment implicates that MFN1, MFN2, and Drp1 are essential for postnatal heart development and metabolic remodeling in human beings and mice [71, 75]. Our findings support the notion that SPD can regulate the IUH-induced imbalance in mitochondrial dynamics by inhibiting fission and promoting fusion, which improves mitochondrial function and alleviating myocardial injury in neonatal rats.

There are some limitations to our study, however. For example, hypoxia exposure during pregnancy often decreases maternal food intake, which results in maternal undernutrition. Reduced nutrient intake during pregnancy is associated with the development of a "thrifty phenotype" in offspring, which leads to an increased prevalence of CVD in adulthood. In the present study, maternal food intake was not quantified; thus, we cannot exclude the effects of maternal undernutrition on the pathophysiological changes seen in newborn hearts. Moreover, we established an animal model of IUH by exposing pregnant rats to hypoxia for $4 \mathrm{~h}$ per day. Indeed, this is a model of chronic intermittent hypoxia $(\mathrm{CIH})$ rather than a chronic hypoxia pregnancy. In clinical studies, $\mathrm{CIH}$ was found to be an important pathophysiological change induced by obstructive sleep apnea-hypopnea syndrome (OSAHS). Evidence shows that OSAHS affects up to onethird of women by the third trimester [76]. OSAHS leads to the upregulation of oxidative stress and pathways of inflammation [77], which are also mechanisms of chronic hypoxia. Finally, it should be mentioned that the PAs include SP, SPD, and their precursor diamine PU. Both SP and SPD can act as direct ROS scavengers, whereas PU exhibits low efficacy in ROS neutralization [63]. In the present study, we showed that maternal SPD supplementation ameliorates mitochondrial function via inhibition of mitochondrial oxidative stress in the hearts of newborns exposed to IUH, but we did not compare the effects of SP, SPD, and PU. In the future, we aim to compare the cardioprotective effects of these PAs on newborn rats exposed to IUH.

\section{Conclusions}

The present study demonstrated that prenatal exposure to hypoxia leads to decreased synthesis and increased breakdown of cardiac PA in neonatal rats. Exogenous SPD administration to hypoxic mothers during pregnancy showed beneficial effects on cardiac fibrosis, cell proliferation and apoptosis, cardiac mitochondrial ROS levels, and mitochondrial fission/fusion balance in the neonatal rats. Our study findings may also aid the development of 
preventative or therapeutic strategies aimed at IUH offspring to prevent adult CVD. However, the effects of prenatal SPD treatment on fetal cardiac development and the pathways that link abnormal changes during fetal development with those in the offspring still need to be addressed in future studies.

\section{Data Availability}

The data used to support the findings of this study are available from the corresponding authors upon request.

\section{Conflicts of Interest}

The authors declare that there is no conflict of interest regarding the publication of this paper.

\section{Authors' Contributions}

Sazonova Elena Nikolaevna and Yajun Zhao contributed equally to this work.

\section{Acknowledgments}

We kindly thank Dr. Weihua Zhang for kindly providing MitoTracker Green and Dr. Li Zhang for her editorial assistance. We are also very grateful to Prof. Changqing $\mathrm{Xu}$ and Yie Tian for their critical reading of this manuscript. This study was supported by grants from the National Natural Science Foundation of China (grant numbers 81170178 and 8160344), the Emergency Management Project of National Natural Science Foundation of China (grant number 31751004), the Natural Science Foundation of Heilongjiang Province (grant number H2016015), and the Postgraduate Research Innovation Fund of Harbin Medical University (grant number YJSCX2014-06HYD).

\section{Supplementary Materials}

The detailed statistical analysis of the comparison between the Hpx-Spd group and the control group, the Hpx-Spd-DFMO group and the control group, and the Hpx-Spd-DFMO group and the Hpx group of all observation items of the newborn rat heart was provided. (Supplementary Materials)

\section{References}

[1] D. A. Giussani, L. Bennet, A. N. Sferruzzi-Perri, O. R. Vaughan, and A. L. Fowden, "Hypoxia, fetal and neonatal physiology: 100 years on from Sir Joseph Barcroft," The Journal of Physiology, vol. 594, no. 5, pp. 1105-1111, 2016.

[2] D. A. Giussani and S. T. Davidge, "Developmental programming of cardiovascular disease by prenatal hypoxia," Journal of Developmental Origins of Health and Disease, vol. 4, no. 5, pp. 328-337, 2013.

[3] P. Zhang, J. Ke, Y. Li et al., "Long-term exposure to high altitude hypoxia during pregnancy increases fetal heart susceptibility to ischemia/reperfusion injury and cardiac dysfunction," International Journal of Cardiology, vol. 274, no. 274, pp. 7-15, 2019.
[4] K. L. Brain, B. J. Allison, Y. Niu et al., "Intervention against hypertension in the next generation programmed by developmental hypoxia," PLOS Biology, vol. 17, no. 1, article e2006552, 2019.

[5] P. Rodríguez-Rodríguez, D. Ramiro-Cortijo, C. G. ReyesHernández, A. L. López de Pablo, M. C. González, and S. M. Arribas, "Implication of oxidative stress in fetal programming of cardiovascular disease," Frontiers in Physiology, vol. 9, article 602, 2018.

[6] D. A. Giussani, E. J. Camm, Y. Niu et al., "Developmental programming of cardiovascular dysfunction by prenatal hypoxia and oxidative stress," PLoS One, vol. 7, no. 2, article e31017, 2012

[7] I. E. Peterside, M. A. Selak, and R. A. Simmons, "Impaired oxidative phosphorylation in hepatic mitochondria in growthretarded rats," American Journal of Physiology-Endocrinology and Metabolism, vol. 285, no. 6, pp. E1258-E1266, 2003.

[8] Y. Y. Lee, H. J. Lee, S. S. Lee et al., "Taurine supplementation restored the changes in pancreatic islet mitochondria in the fetal protein-malnourished rat," British Journal of Nutrition, vol. 106, no. 8, pp. 1198-1206, 2011.

[9] Y. M. Al-Hasan, G. A. Pinkas, and L. P. Thompson, "Prenatal hypoxia reduces mitochondrial protein levels and cytochrome c oxidase activity in offspring guinea pig hearts," Reproductive Sciences, vol. 21, no. 7, pp. 883-891, 2014.

[10] J. Marín-García and A. T. Akhmedov, "Mitochondrial dynamics and cell death in heart failure," Heart Failure Reviews, vol. 21, no. 2, pp. 123-136, 2016.

[11] M. Song, K. Mihara, Y. Chen, L. Scorrano, and G. W. Dorn II, "Mitochondrial fission and fusion factors reciprocally orchestrate mitophagic culling in mouse hearts and cultured fibroblasts," Cell Metabolism, vol. 21, no. 2, pp. 273-286, 2015.

[12] K. N. Papanicolaou, R. J. Khairallah, G. A. Ngoh et al., "Mitofusin-2 maintains mitochondrial structure and contributes to stress-induced permeability transition in cardiac myocytes," Molecular and Cellular Biology, vol. 31, no. 6, pp. 1309-1328, 2011.

[13] T. M. Stewart, T. T. Dunston, P. M. Woster, and R. A. Casero Jr, "Polyamine catabolism and oxidative damage," Journal of Biological Chemistry, vol. 293, no. 48, pp. 18736-18745, 2018.

[14] T. Thomas and T. J. Thomas, "Polyamines in cell growth and cell death: molecular mechanisms and therapeutic applications," Cellular and Molecular Life Sciences, vol. 58, no. 2, pp. 244-258, 2001.

[15] J. W. Jeong, H. J. Cha, M. H. Han et al., "Spermidine protects against oxidative stress in inflammation models using macrophages and zebrafish," Biomolecules \& Therapeutics, vol. 26, no. 2, pp. 146-156, 2018.

[16] T. Zou, J. N. Rao, X. Guo et al., "NF- $\kappa$ B-mediated IAP expression induces resistance of intestinal epithelial cells to apoptosis after polyamine depletion," American Journal of PhysiologyCell Physiology, vol. 286, no. 5, pp. C1009-C1018, 2004.

[17] J. E. Rider, A. Hacker, C. A. Mackintosh, A. E. Pegg, P. M. Woster, and R. A. Casero Jr, "Spermine and spermidine mediate protection against oxidative damage caused by hydrogen peroxide," Amino Acids, vol. 33, no. 2, pp. 231-240, 2007.

[18] N. Minois, D. Carmona-Gutierrez, M. A. Bauer et al., "Spermidine promotes stress resistance in Drosophila melanogaster through autophagy-dependent and -independent pathways," Cell Death \& Disease, vol. 3, no. 10, article e401, 2012. 
[19] T. Eisenberg, H. Knauer, A. Schauer et al., "Induction of autophagy by spermidine promotes longevity," Nature Cell Biology, vol. 11, no. 11, pp. 1305-1314, 2009.

[20] F. Gaboriau, M. Vaultier, J. P. Moulinoux, and J. G. Delcros, "Antioxidative properties of natural polyamines and dimethylsilane analogues," Redox Report, vol. 10, no. 1, pp. 9-18, 2005.

[21] H. Kwon, G. Wu, F. W. Bazer, and T. E. Spencer, "Developmental changes in polyamine levels and synthesis in the ovine conceptus," Biology of Reproduction, vol. 69, no. 5, pp. 16261634, 2003.

[22] G. Wu, F. W. Bazer, M. C. Satterfield et al., "Impacts of arginine nutrition on embryonic and fetal development in mammals," Amino Acids, vol. 45, no. 2, pp. 241-256, 2013.

[23] I.-H. Park and M.-M. Kim, "Inhibitory effect of spermidine with antioxidant activity on oxidative stress in human dermal fibroblasts," Journal of Life Science, vol. 21, no. 5, pp. 693-699, 2011.

[24] H. C. Ha, N. S. Sirisoma, P. Kuppusamy, J. L. Zweier, P. M. Woster, and R. A. Casero, "The natural polyamine spermine functions directly as a free radical scavenger," Proceedings of the National Academy of Sciences, vol. 95, no. 19, pp. 1114011145, 1998.

[25] K. C. Das and H. P. Misra, "Hydroxyl radical scavenging and singlet oxygen quenching properties of polyamines," Molecular and Cellular Biochemistry, vol. 262, no. 1/2, pp. 127-133, 2004.

[26] I. G. Sava, V. Battaglia, C. A. Rossi, M. Salvi, and A. Toninello, "Free radical scavenging action of the natural polyamine spermine in rat liver mitochondria," Free Radical Biology and Medicine, vol. 41, no. 8, pp. 1272-1281, 2006.

[27] M. P. Rigobello, A. Toninello, D. Siliprandi, and A. Bindoli, "Effect of spermine on mitochondrial glutathione release," Biochemical and Biophysical Research Communications, vol. 194, no. 3, pp. 1276-1281, 1993.

[28] Y. Zhao, W. Zhang, and Y. Wang, "Effect of ornithine decarboxylase/polyamine system on ischemic preconditioning myocardial protection in rats," Chinese Journal of Pathophysiology, vol. 25, no. 12, pp. 2295-2301, 2009.

[29] W. Wang, H. Zhang, G. Xue et al., "Exercise training preserves ischemic preconditioning in aged rat hearts by restoring the myocardial polyamine pool," Oxidative Medicine and Cellular Longevity, vol. 2014, Article ID 457429, 14 pages, 2014.

[30] C. M. Herring, F. W. Bazer, G. A. Johnson, and G. Wu, "Impacts of maternal dietary protein intake on fetal survival, growth, and development," Experimental Biology and Medicine, vol. 243, no. 6, pp. 525-533, 2018.

[31] F. Madeo, T. Eisenberg, F. Pietrocola, and G. Kroemer, "Spermidine in health and disease," Science, vol. 359, no. 6374, article eaan2788, 2018.

[32] T. Eisenberg, M. Abdellatif, S. Schroeder et al., "Cardioprotection and lifespan extension by the natural polyamine spermidine," Nature Medicine, vol. 22, no. 12, pp. 1428-1438, 2016.

[33] H. G. Frohnhöfer, S. Geiger-Rudolph, M. Pattky et al., "Spermidine, but not spermine, is essential for pigment pattern formation in zebrafish," Biology Open, vol. 5, no. 6, pp. 736$744,2016$.

[34] S. Jamwal and P. Kumar, "Spermidine ameliorates 3nitropropionic acid (3-NP)-induced striatal toxicity: possible role of oxidative stress, neuroinflammation, and neurotransmitters," Physiology \& Behavior, vol. 155, no. 1, pp. 180-187, 2016.
[35] M. Chrisam, M. Pirozzi, S. Castagnaro et al., "Reactivation of autophagy by spermidine ameliorates the myopathic defects of collagen VI-null mice," Autophagy, vol. 11, no. 12, pp. 2142-2152, 2015.

[36] Q. Xue and L. Zhang, "Prenatal hypoxia causes a sexdependent increase in heart susceptibility to ischemia and reperfusion injury in adult male offspring: role of protein kinase $C$ epsilon," Journal of Pharmacology and Experimental Therapeutics, vol. 330, no. 2, pp. 624-632, 2009.

[37] F. Chen, S. du, J. Bian, Z. B. You, and Y. Wu, "Chronic hypoxia exposure during pregnancy is associated with a decreased active nursing activity in mother and an abnormal birth weight and postnatal growth in offspring of rats," Hormones and Behavior, vol. 61, no. 4, pp. 504-511, 2012.

[38] G. Li, Y. Xiao, J. L. Estrella, C. A. Ducsay, R. D. Gilbert, and L. Zhang, "Effect of fetal hypoxia on heart susceptibility to ischemia and reperfusion injury in the adult rat," Journal of the Society for Gynecologic Investigation, vol. 10, no. 5, pp. 265-274, 2003.

[39] Z. Jin, W. Zhang, H. Yang et al., "Maternal treatment with agonistic autoantibodies against type-1 angiotensin II receptor in late pregnancy increases apoptosis of myocardial cells and myocardial susceptibility to ischemia-reperfusion injury in offspring rats," PLoS One, vol. 8, no. 11, article e80709, 2013.

[40] H. Li, C. Wei, J. Gao et al., "Mediation of dopamine D2 receptors activation in post-conditioning-attenuated cardiomyocyte apoptosis," Experimental Cell Research, vol. 323, no. 1, pp. 118-130, 2014.

[41] Q. Zhang, Y. Deng, W. Lai et al., "Maternal inflammation activated ROS-p38 MAPK predisposes offspring to heart damages caused by isoproterenol via augmenting ROS generation," Scientific Reports, vol. 6, no. 1, 2016.

[42] Y. Hiraumi, E. Iwai-Kanai, S. Baba et al., "Granulocyte colonystimulating factor protects cardiac mitochondria in the early phase of cardiac injury," American Journal of PhysiologyHeart and Circulatory Physiology, vol. 296, no. 3, pp. H823H832, 2009.

[43] L. C. Evans, H. Liu, and L. P. Thompson, "Differential effect of intrauterine hypoxia on caspase 3 and DNA fragmentation in fetal guinea pig hearts and brains," Reproductive Sciences, vol. 19, no. 3, pp. 298-305, 2012.

[44] J. C. Sun, B. Liu, R. W. Zhang et al., "Overexpression of Barrestin1 in the rostral ventrolateral medulla downregulates angiotensin receptor and lowers blood pressure in hypertension," Frontiers in Physiology, vol. 9, no. 3, pp. 298-305, 2018.

[45] M. Liu, G. Shi, K.-C. Yang et al., "Role of protein kinase C in metabolic regulation of the cardiac $\mathrm{Na}+$ channel," Heart Rhythm., vol. 14, no. 3, pp. 440-447, 2017.

[46] M. Reers, S. T. Smiley, C. Mottola-Hartshorn, A. Chen, M. Lin, and L. B. Chen, "[29] Mitochondrial membrane potential monitored by JC-1 dye," Methods in Enzymology, vol. 260, no. 1, pp. 406-417, 1995.

[47] M. T. Neary, K. E. Ng, M. H. R. Ludtmann et al., "Hypoxia signaling controls postnatal changes in cardiac mitochondrial morphology and function," Journal of Molecular and Cellular Cardiology, vol. 74, pp. 340-352, 2014.

[48] Y. Y. Lenis, M. A. Elmetwally, J. G. Maldonado-Estrada, and F. W. Bazer, "Physiological importance of polyamines," Zygote, vol. 25, no. 3, pp. 244-255, 2017. 
[49] P. L. C. Lefèvre, M.-F. Palin, and B. D. Murphy, "Polyamines on the reproductive landscape," Endocrine Reviews, vol. 32, no. 5, pp. 694-712, 2011.

[50] S. Guha and J. Janne, "The synthesis and accumulation of polyamines in reproductive organs of the rat during pregnancy," Biochimica et Biophysica Acta (BBA) - General Subjects, vol. 437, no. 1, pp. 244-252, 1976.

[51] L. D. Brown, A. S. Green, S. W. Limesand, and P. J. Rozance, "Maternal amino acid supplementation for intrauterine growth restriction," Frontiers in Bioscience, vol. S3, no. 2, pp. 428-444, 2011.

[52] O. Basystyi, "Polyamine concentration in erythrocytes in the blood of pregnant women with intrauterine growth retardation," Georgian medical news, vol. 1, no. 261, pp. 22-26, 2016.

[53] A. Casti, B. Salvadori, N. Reali, B. Ferrari, G. Orlandini, and A. Corti, "Concentrations of polyamines in maternal and umbilical-cord blood and in the amniotic fluid during normal human pregnancy and in feto-placental insufficiency," Acta Bio-medica del'Ateneo Parmense, vol. 50, no. 1, pp. 15-26, 1979.

[54] S. Gong, U. Sovio, I. L. M. H. Aye et al., "Placental polyamine metabolism differs by fetal sex, fetal growth restriction, and preeclampsia," JCI Insight, vol. 3, no. 13, article 120723, 2018.

[55] W. Tong, Q. Xue, Y. Li, and L. Zhang, "Maternal hypoxia alters matrix metalloproteinase expression patterns and causes cardiac remodeling in fetal and neonatal rats," American Journal of Physiology-Heart and Circulatory Physiology, vol. 301, no. 5, pp. H2113-H2121, 2011.

[56] E. H. Yeung, C. Robledo, N. Boghossian, C. Zhang, and P. Mendola, "Developmental origins of cardiovascular disease," Current Epidemiology Reports, vol. 1, no. 1, pp. 916, 2014.

[57] R. J. Vosatka, P. M. Hassoun, and K. B. Harvey-Wilkes, "Dietary L-arginine prevents fetal growth restriction in rats," American Journal of Obstetrics and Gynecology, vol. 178, no. 2, pp. 242-246, 1998.

[58] H. G. Gumus, M. Illa, L. Pla, M. Zamora, F. Crispi, and E. Gratacos, "Nutritional intra-amniotic therapy increases survival in a rabbit model of fetal growth restriction," PLoS One, vol. 13, no. 2, article e0193240, 2018.

[59] R. Kibe, S. Kurihara, Y. Sakai et al., "Upregulation of colonic luminal polyamines produced by intestinal microbiota delays senescence in mice," Scientific Reports, vol. 4, no. 1, 2014.

[60] M. Matsumoto, S. Kurihara, R. Kibe, H. Ashida, and Y. Benno, "Longevity in mice is promoted by probiotic-induced suppression of colonic senescence dependent on upregulation of gut bacterial polyamine production," PLoS One, vol. 6, no. 8, article e23652, 2011.

[61] J. R. Fozard, M. L. Part, N. J. Prakash, and J. Grove, "Inhibition of murine embryonic development by alpha-difluoromethylornithine, an irreversible inhibitor of ornithine decarboxylase," European Journal of Pharmacology, vol. 65, no. 4, pp. 379-391, 1980.

[62] B. A. O'Toole, K. W. Huffman, and J. P. Gibson, "Effects of eflornithine hydrochloride (DFMO) on fetal development in rats and rabbits," Teratology, vol. 39, no. 2, pp. 103-113, 1989.

[63] H. M. Wallace and A. V. Fraser, "Inhibitors of polyamine metabolism: review article," Amino Acids, vol. 26, no. 4, pp. 353-365, 2004.

[64] M. M. Aljunaidy, J. S. Morton, C.-L. M. Cooke, and S. T. Davidge, "Prenatal hypoxia and placental oxidative stress: linkages to developmental origins of cardiovascular disease," American Journal of Physiology-Regulatory, Integrative and Comparative Physiology, vol. 313, no. 4, pp. R395-R399, 2017.

[65] V. Petronilli, D. Penzo, L. Scorrano, P. Bernardi, and F. di Lisa, "The mitochondrial permeability transition, release of cytochromecand cell death," Journal of Biological Chemistry, vol. 276, no. 15, pp. 12030-12034, 2001.

[66] S. L. Bourque, V. W. Dolinsky, J. R. B. Dyck, and S. T. Davidge, "Maternal resveratrol treatment during pregnancy improves adverse fetal outcomes in a rat model of severe hypoxia," Placenta, vol. 33, no. 5, pp. 449-452, 2012.

[67] V. H. Parraguez, S. Mamani, E. Cofré et al., "Disturbances in maternal steroidogenesis and appearance of intrauterine growth retardation at high-altitude environments are established from early pregnancy. Effects of treatment with antioxidant vitamins," PLoS One, vol. 10, no. 11, article e0140902, 2015.

[68] N. Itani, K. L. Skeffington, C. Beck, Y. Niu, and D. A. Giussani, "Melatonin rescues cardiovascular dysfunction during hypoxic development in the chick embryo," ournal of Pineal Research, vol. 60, no. 1, pp. 16-26, 2016.

[69] L. M. Reyes, J. S. Morton, R. Kirschenman, D. S. DeLorey, and S. T. Davidge, "Vascular effects of aerobic exercise training in rat adult offspring exposed to hypoxia-induced intrauterine growth restriction," The Journal of Physiology, vol. 593, no. 8, pp. 1913-1929, 2015.

[70] G. W. Dorn, "Mitochondrial dynamism and heart disease: changing shape and shaping change," EMBO Molecular Medicine, vol. 7, no. 7, pp. 865-877, 2015.

[71] T. Ishihara, R. Ban-Ishihara, M. Maeda et al., "Dynamics of mitochondrial DNA nucleoids regulated by mitochondrial fission is essential for maintenance of homogeneously active mitochondria during neonatal heart development," Molecular and Cellular Biology, vol. 35, no. 1, pp. 211223, 2014.

[72] G. D. Lopaschuk and J. S. Jaswal, "Energy metabolic phenotype of the cardiomyocyte during development, differentiation, and postnatal maturation," Journal of Cardiovascular Pharmacology, vol. 56, no. 2, pp. 130-140, 2010.

[73] O. J. Martin, L. Lai, M. M. Soundarapandian et al., “A role for peroxisome proliferator-activated receptor $\gamma$ coactivator- 1 in the control of mitochondrial dynamics during postnatal cardiac growth," Circulation Research, vol. 114, no. 4, pp. 626-636, 2014.

[74] G. W. Dorn II, R. B. Vega, and D. P. Kelly, "Mitochondrial biogenesis and dynamics in the developing and diseased heart," Genes and Developmen, vol. 29, no. 19, pp. 19811991, 2015.

[75] K. N. Papanicolaou, R. Kikuchi, G. A. Ngoh et al., "Mitofusins 1 and 2 are essential for postnatal metabolic remodeling in heart," Circulation Research, vol. 111, no. 8, pp. 1012-1026, 2012.

[76] S. M. Johnson, K. S. Randhawa, J. J. Epstein et al., "Gestational intermittent hypoxia increases susceptibility to neuroinflammation and alters respiratory motor control in neonatal rats," Respiratory Physiology \& Neurobiology, vol. 256, no. 1, pp. 128-142, 2018.

[77] T. DeMartino, R. E. Ghoul, L. Wang et al., "Oxidative stress and inflammation differentially elevated in objective versus habitual subjective reduced sleep duration in obstructive sleep apnea," Sleep, vol. 39, no. 7, pp. 1361-1369, 2016. 


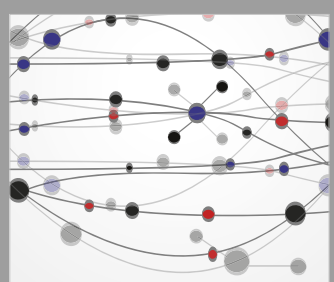

The Scientific World Journal
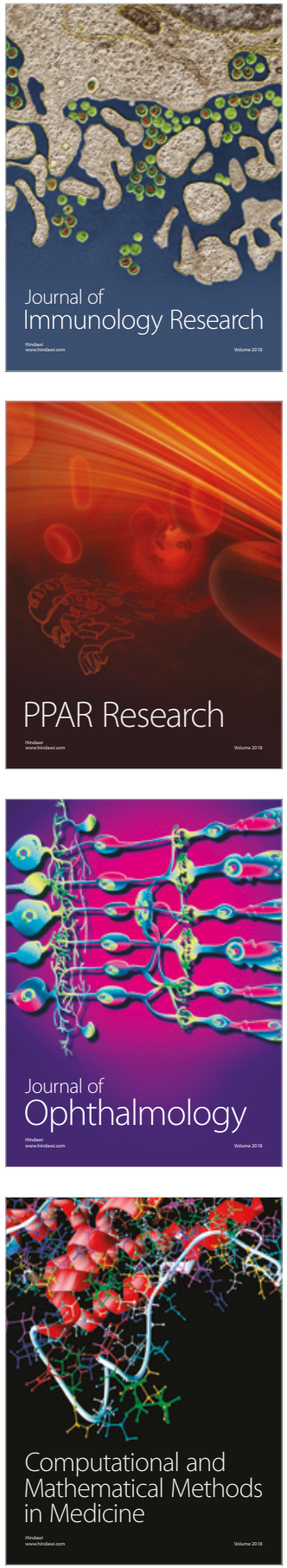

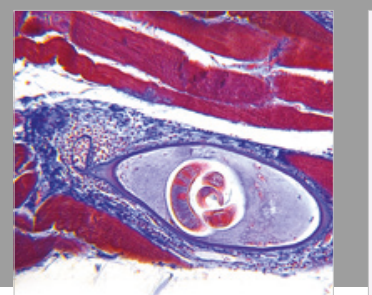

Gastroenterology Research and Practice

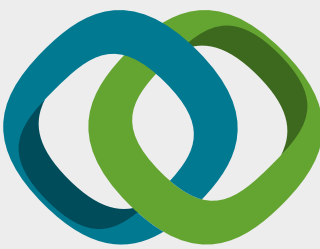

\section{Hindawi}

Submit your manuscripts at

www.hindawi.com
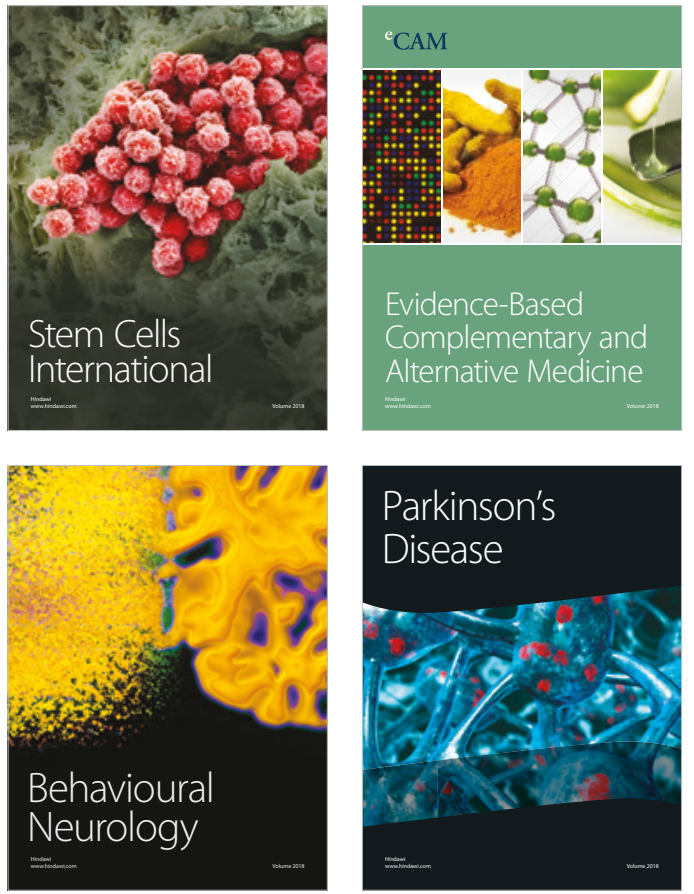

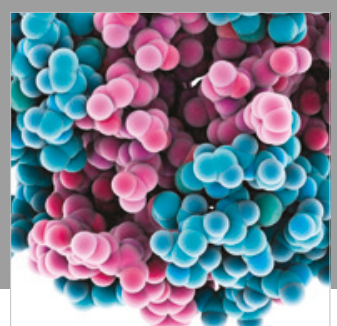

ournal of

Diabetes Research

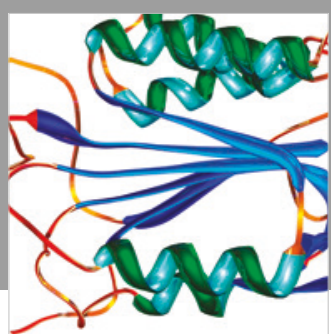

Disease Markers
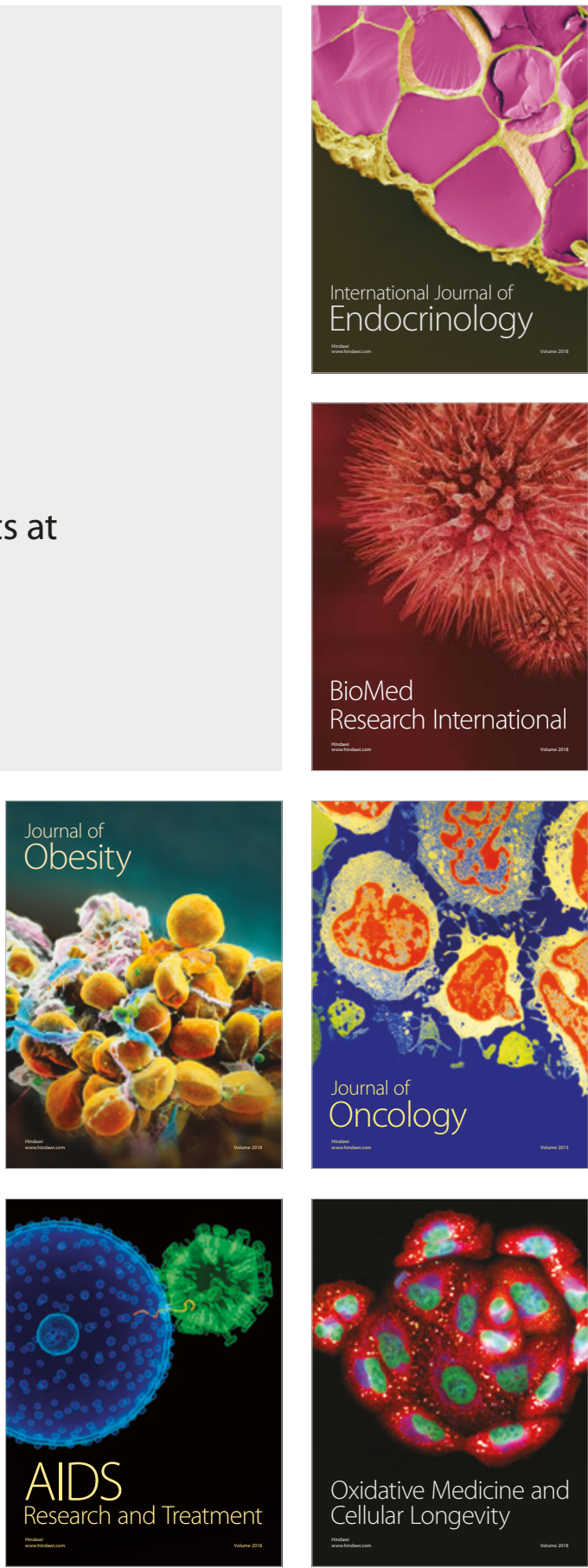